\title{
Relic density of dark matter in the inert doublet model beyond leading order for the low mass region. II. Coannihilation
}

\author{
Shankha Banerjee $\odot,{ }^{1, *}$ Fawzi Boudjema $\oplus^{2, \dagger}$ Nabarun Chakrabarty, ${ }^{3,4, \$}$ and Hao Sun ${ }^{5, \S}$ \\ ${ }^{1}$ CERN, Theoretical Physics Department, CH-1211 Geneva 23, Switzerland \\ ${ }^{2}$ LAPTh, Université Savoie Mont Blanc, CNRS, BP 110, F-74941 Annecy-le-Vieux, France \\ ${ }^{3}$ Centre for High Energy Physics, Indian Institute of Science, C.V. Raman Avenue, Bangalore 560012, India \\ ${ }^{4}$ Department of Physics, Indian Institute of Technology Kanpur, Kanpur, Uttar Pradesh 208016, India \\ ${ }^{5}$ Institute of Theoretical Physics, School of Physics, Dalian University of Technology, \\ Dalian 116024, People's Republic of China
}

(Received 20 February 2021; accepted 13 August 2021; published 5 October 2021)

\begin{abstract}
We examine the relic density of the light mass dark matter region in the inert doublet model (IDM) when the dominant process is due to coannihilation between the lightest neutral scalars of the model. The full oneloop electroweak corrections are computed in an on shell scheme and are found to be well approximated as an effective cross section expressed in terms of $Z$ observables. The electroweak corrections to the subdominant process, which consists of an annihilation into an on shell $W$ and an off shell $W$, that is calculated as a annihilation into a three-body final state, is also performed. In the calculation of the latter in this case of coannihilation, although the SM Higgs exchange is absent, it is induced at one loop, but the cross section does not show a peak behavior despite the crossing of the Higgs resonance. This cross section reveals an important dependence on a parameter that describes the self-interaction of the new scalars (solely within the dark sector), a parameter which is not accessible in tree-level calculations of standard model-IDM interactions.
\end{abstract}

DOI: $10.1103 /$ PhysRevD.104.075003

\section{INTRODUCTION}

In the very thorough analysis we have conducted in the parent paper [1] of this series on the available parameter space of the inert doublet model (IDM) $[2,3]$ for the low mass dark matter (DM) (below the $W$ mass, $M_{W}$ ), we have confirmed the survival of a very small region with a DM mass of 55-60 GeV, which has been unraveled [4,5] only recently. Within the freeze-out mechanism this is possible, mainly, through efficient coannihilation (see Fig. 1). The two additional (lightest) neutral scalars of the IDM, $X$ and $A$, need to be thermodynamically close to one another for this coannihilation to take place. In other words, this requires a certain degree of mass degeneracy which, in the context of the IDM, is not necessarily fine-tuned and unnatural. The $Z$-mediated coannihilation is into the (light) fermion pairs, not including the top quark, of the Standard Model (SM). The process is a gauge interaction induced process, and as

\footnotetext{
*shankha.banerjee@cern.ch

†boudjema@lapth.cnrs.fr

*chakrabartynabarun@gmail.com

§haosun@dlut.edu.cn
}

Published by the American Physical Society under the terms of the Creative Commons Attribution 4.0 International license. Further distribution of this work must maintain attribution to the author(s) and the published article's title, journal citation, and DOI. Funded by SCOAP. such, it is quite efficient. So efficient in fact that to obtain a value of the relic density around 0.12 , the coannihilation cross sections must be dampened by the Boltzmann factor, $e^{-m_{-} / T}$, where $m_{-}=M_{A}-M_{X}$ is the mass splitting and $T$ is the temperature. The Boltzmann factor in the thermally averaged cross sections should furnish enough reduction to counterbalance the large cross sections. This in turn requires the mass splitting to be not too small. In our analyses, we found that $m_{-}=8 \mathrm{GeV}$ is an optimal value. This value is at the edge of the LEPII constraint on the IDM. Higher values of the mass splitting would lead to visible tracks at LEPII that disqualify the model while lower values do not give enough Boltzmann reduction leading to too small $\Omega h^{2}$. For our one-loop analyses, we have judiciously retained two such benchmarks points with mass $M_{X}=58,60 \mathrm{GeV}$ and $m_{-}=8 \mathrm{GeV}$; see Table I.

As we will see, this $2 \mathrm{GeV}$ difference in the DM mass between the two benchmarks is enough to give a $20 \%$ reduction in the (co)annihilating cross sections in P60 compared to P58. While the Boltzmann factor is (slightly) larger for the $M_{X}=60 \mathrm{GeV}$ case to somehow compensate for the drop in the $A X \rightarrow f \bar{f}$ cross sections, what really benefits P60 is a larger cross section of the annihilating process $X X \rightarrow W W^{\star}$, thanks to a larger phase space than in the case of $M_{X}=58 \mathrm{GeV}$. The masses of all the scalars of the IDM have been set to define the model. An extra parameter that is needed to fully describe the interaction 
TABLE I. Characteristics of the coannihilation benchmarks points P58 and P60. All masses are in GeV. Here, we take $\lambda_{L}=0$. We also show tree-level [calculated with $(\alpha(0)$ ] relic density and the weight in percent of each channel contributing to the relic density. $f$ stands for all light fermions in the SM (the top-quark channel is closed). For more precise numbers, especially of the underlying parameters $\lambda_{3,4,5}$, please refer to Ref. [1].

\begin{tabular}{lcc}
\hline \hline & P58 & P60 \\
\hline$M_{X}$ & 58 & 60 \\
$M_{A}, M_{H^{ \pm}}$ & 66,110 & 68,150 \\
$\left(\lambda_{3}, \lambda_{4}, \lambda_{5}\right)$ & $(0.28,-0.26,-0.02)$ & $(0.60,-0.58,-0.02)$ \\
$\alpha(0)$ & \multicolumn{2}{c}{$\Omega h^{2}$} \\
$\Omega_{W W^{\star}}(\%)$ & 0.113 & 0.116 \\
$\Omega_{A X \rightarrow \sum f \bar{f}}(\%)$ & 5 & 9 \\
\hline \hline
\end{tabular}

between these scalar and the SM scalar sector is $\lambda_{L}$. In fact, $\lambda_{L}$ describes the interaction strength of the SM Higgs boson to the DM, $X$, see [1] for details. We find that in order to keep the cross sections small, including $X X$ annihilations, $\lambda_{L}$ must be as small as possible. This is the reason that for the surviving points, we take $\lambda_{L}=0$. This is a judicious choice not only since the phenomenology requires it but it also serves, for P58, to concentrate solely on the coannihilation process (which represents $95 \%$ of the contribution to the relic density) and extract its dominant contribution in an analytic form. This is not the only reason. While in both P58 and P60 $\lambda_{L}=0$ makes $X X \rightarrow h \rightarrow b \bar{b}, W W^{\star}$ vanish, P60 has a non-negligible (about 10\%) contribution from $X X \rightarrow W W^{\star}$ even with $\lambda_{L}=0$. Therefore, the tree-level process does not feel the SM Higgs resonance; however, at one loop, an induced $h X X$ coupling develops. Yet the amplitude is not resonant when it crosses the Higgs mass as we will explain. This scenario is therefore a good introduction to both the continuum nonresonant $X X \rightarrow W W^{\star}$ and the Higgs resonance benchmarks. Both deserve dedicated analyses. In the next section, we concentrate on the electroweak radiative corrections for the coannihilation process $A X \rightarrow f \bar{f}$. We then turn to the subdominant $X X \rightarrow W W^{\star}$ process, which can be of relevance for this region. In the present paper, we include the results of the one-loop contribution to $X X \rightarrow W W^{\star} \equiv W f \bar{f}^{\prime}$, but we leave many of the technical details covering both $2 \rightarrow 3$ processes at one loop and the treatment of the SM Higgs resonance to more detailed presentations in Refs. [6,7].

\section{THE COANNIHILATION CROSS SECTION $A X \rightarrow f \bar{f}$}

\section{A. $A X \rightarrow f \bar{f}$ : Tree-level considerations}

The coannihilation cross sections are completely determined by the gauge coupling, given the masses of $X$ and $A$. The annihilations are essentially $P$ wave with a very small $S$-wave contribution that is noticeable only for extremely small velocities in the case of the heaviest final state fermion, the $b$ quark. For such $2 \rightarrow 2$ processes, the cross section can be described by a transparent analytical formula. With $m_{f}$, the mass of the final fermion, the tree-level cross section can be written as

$$
\sigma_{A X \rightarrow f \bar{f}} v=\sigma_{v^{2}}^{f \bar{f}} v^{2}+\frac{m_{f}^{2}}{s} \sigma_{0}^{f \bar{f}}
$$

The $P$ wave, $v^{2}$ term, dominates by far while the very small $S$-wave contribution, $\sigma_{0}$, is suppressed by the chiral factor $m_{f}^{2}$. The latter is further suppressed by the mass splitting factor, $m_{-}^{2}$, as shown explicitly below. With the "running" partial width,

$$
\begin{aligned}
& \Gamma_{Z \rightarrow f \bar{f}}(s)=N_{c}^{f} \frac{g^{2} \sqrt{s}}{12 \pi c_{W}^{2}} \beta_{f}\left(g_{V}^{2} \frac{3-\beta_{f}^{2}}{2}+g_{A}^{2} \beta_{f}^{2}\right) \\
& \underset{s \gg m_{f}^{2}}{\simeq} \frac{\sqrt{s}}{M_{Z}} \Gamma_{Z \rightarrow f \bar{f}}, \quad N_{c} \text { is the color factor, } \\
& \text { and } \beta_{f}=\sqrt{1-\frac{4 m_{f}^{2}}{s}}, \quad g_{V}=\frac{T_{3 f}}{2}-Q_{f} s_{W}^{2}, \\
& g_{A}=\frac{T_{3 f}}{2} \quad\left(T_{3 f}= \pm 1 / 2\right), \\
& \Gamma_{Z \rightarrow f \bar{f}} \equiv N_{c}^{f} \frac{g^{2} M_{Z}}{12 \pi c_{W}^{2}}\left(g_{V}^{2}+g_{A}^{2}\right),
\end{aligned}
$$

( $Q_{f}$ is the charge of the fermion), we have

$$
\begin{aligned}
\sigma_{v^{2}}^{f \bar{f}} & =\frac{g^{2}}{c_{W}^{2}} \frac{1}{8 s} \frac{1-m_{-}^{2} m_{+}^{2} / s^{2}}{\left(1-M_{Z}^{2} / s\right)^{2}} \frac{\Gamma_{Z \rightarrow f \bar{f}}(s)}{\sqrt{s}} \\
& \simeq \frac{g^{2}}{c_{W}^{2}} \frac{1}{8 s} \frac{1-m_{-}^{2} m_{+}^{2} / s^{2}}{\left(1-M_{Z}^{2} / s\right)^{2}} \frac{\Gamma_{Z \rightarrow f \bar{f}}}{M_{Z}} \\
\sigma_{0}^{f \bar{f}} & =\frac{m_{-}^{2} m_{+}^{2}}{s^{2}-m_{-}^{2} m_{+}^{2}} \frac{g^{2}}{c_{W}^{2}} \frac{3}{2 s} \frac{1}{\left(1-M_{Z}^{2} / s\right)^{2}} \frac{\beta_{f} \Gamma_{Z \rightarrow \nu \bar{\nu}}}{M_{Z}} \\
& \simeq \frac{m_{-}^{2} m_{+}^{2}}{s^{2}-m_{-}^{2} m_{+}^{2}} \frac{g^{2}}{c_{W}^{2}} \frac{3}{2 s} \frac{1}{\left(1-M_{Z}^{2} / s\right)^{2}} \frac{\Gamma_{Z \rightarrow \nu \bar{\nu}}}{M_{Z}} .
\end{aligned}
$$

The approximation $(\simeq)$ in Eq. (2.3) which amounts to $m_{f} \rightarrow 0$, is excellent for all fermions, including the bottom quark (for which the approximation is off by about 3 permille for the range of velocities relevant for the calculation of the relic density contributed by these channels). Despite our $P$-wave appellation, note that the $s$ (and hence, $v$ ) dependence contained in $\sigma_{v^{2}}^{f \bar{f}}$ is not small for the two scenarios that we are studying; see Fig. 2. This $s$-dependent factor in the expression of $\sigma_{v^{2}}^{f \bar{f}}$ explains that for the same value of the relative velocity, the coannihilation processes are about 20\% larger for P58 as compared to P60. 


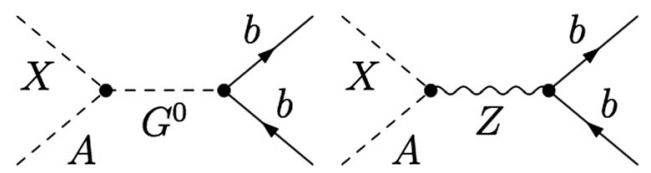

FIG. 1. Tree-level Feynman diagrams (in the nonlinear Feynman gauge $[1,8])$ for $A X \rightarrow b \bar{b}$ as a representative of $A X \rightarrow f \bar{f}$.

Figure 2, which is the result of a full one-loop calculation along the lines detailed in the parent paper [1], exhibits the $P$-wave nature of the coannihilation and confirms the analytical results. Departure from a pure $v^{2}$ dependence is clearly seen. The ratio $\sigma(b \bar{b}) / \sigma(d \bar{d})$ clearly shows the very small contribution of the $S$ wave for small $v$ and the tiny effect of the mass of the fermion for larger $v$. In the case of massless fermions, Fig. 2 confirms that $\sigma(\nu \bar{\nu}) / \sigma(d \bar{d})$ is given by the ratio of the respective partial widths of the $Z$ into these fermions.

\section{B. $A X \rightarrow f \bar{f}$ : Cross sections at one loop}

Since these coannihilation processes do not depend on $\lambda_{L}$, a fully OS renormalization exactly along the lines of the renormalization of the SM can be carried out. The one-loop corrections will have no scale dependence. These processes allow us to test whether the use of a running $\alpha$ is a good approximation for the full one-loop corrections. We show results for all light flavors $f=\nu, l, u, d, b$. For definiteness, we take $\nu=\nu_{\tau}$, and $l=\mu$. We look at both $d$ and $b$ since the latter is affected by the heavy top loop, as is the case for the $Z \rightarrow b \bar{b}$ decay and also because it may be more sensitive to the $\lambda_{2}$ dependence, which appears in the $S$ channel. Recall that $\lambda_{2}$ measures the self-interaction exclusively within the dark sector; see Ref. [1]. We find however

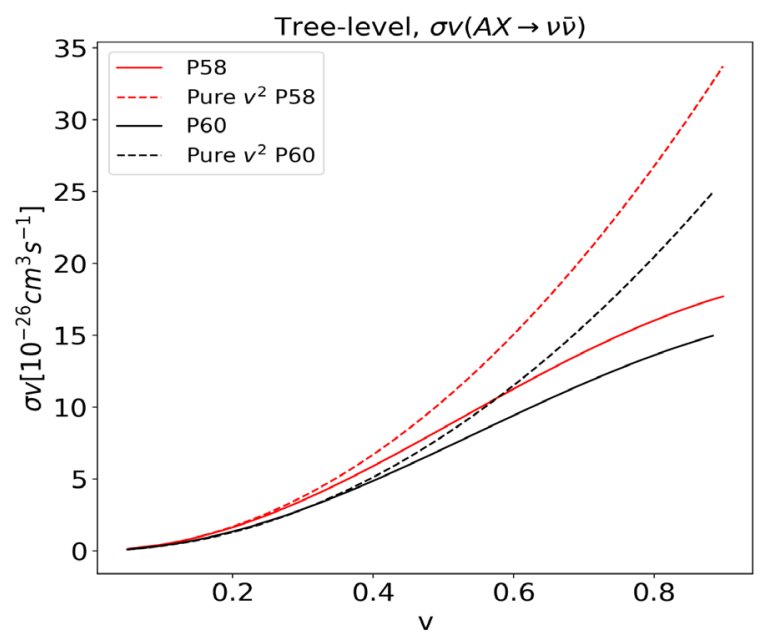

that this $\lambda_{2}$ dependence is very much suppressed by the mass of the (very light) fermions; see Fig. 3, as we explain below.

We perform the full one-loop electroweak corrections for both benchmarks P58 and P60. The electroweak corrections, expressed in terms of relative corrections, are practically exactly the same for all channels for both benchmark points. Therefore, we only show the results for P58. The computation of the radiative corrections for the charged fermions includes the QED final state radiation that we obtain through the slicing technique; see [9], for example. For a fermion of charge $Q_{f}$, these final state QED corrections amount to a relative correction $3 Q_{f}^{2} \alpha / 4 \pi$, which are very small, $<0.2 \%$ (for charged leptons). The QCD final state correction, $\alpha_{s}\left(M_{Z}^{2}\right) / \pi$, amounts to about $3.6 \%$. We have not included the latter in Table II.

Apart from the $b$ quarks, the relative corrections for all fermion final states are practically a modest rescaling of the tree-level cross section. Indeed, the relative corrections are velocity independent. For the $b$ quark, this is also the case for all $v$ but $v \sim 0$, where the $S$ wave contributes negligibly. We will come back to this feature. The flavor dependence of the correction is also very small, all corrections being within $2 \%$ of each other. Apart from the behavior at $v \sim 0$, the corrections at all $v$ for the bottom-quark final states are slightly different due to the contribution of the heavy top quark, a feature known from $Z$ physics. On average, the corrections are about $6 \%$ and are therefore a factor 2 lower than if we had used $\alpha\left(M_{Z}^{2}\right)$. Genuine box and triangle contributions are therefore not negligible.

Inspired by Eq. (2.3), and in view that the $S$-wave contribution is tiny even in the case of $b \bar{b}$, we suggest to improve the tree-level cross sections by using

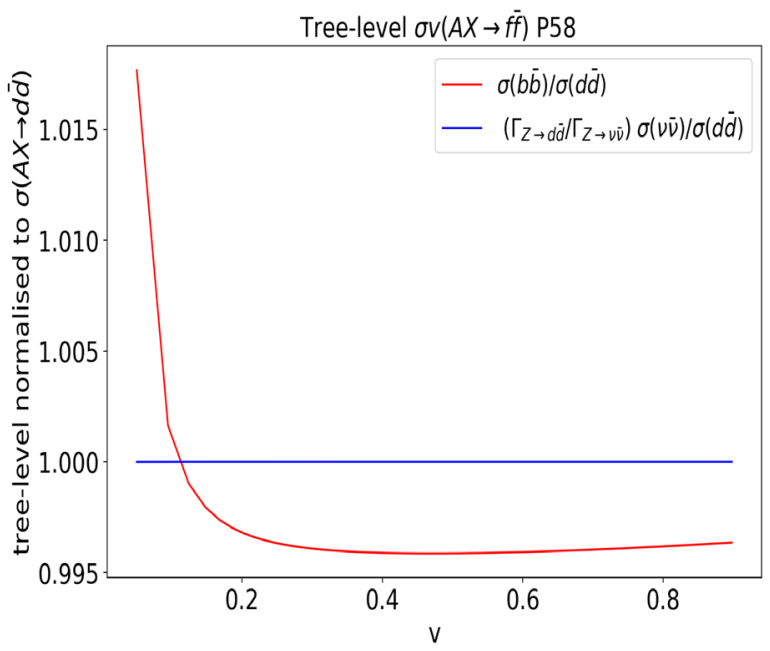

FIG. 2. Left panel: The tree-level cross section for $\sigma(X X \rightarrow \nu \bar{\nu})$ for P58 and P60 as a function of the relative velocity. The dashed curves are the pure $P$-wave $\left(\propto v^{2}\right)$ approximation. We see that this pure $v^{2}$ approximation is only valid for $v<0.4$. Note that the cross sections are quite large; however, remember that a Boltzmann suppression will be applied to these coannihilations cross sections when they are converted to effective velocity/temperature averaged cross sections. Right panel: The $b \bar{b}$ cross section normalised to the $d \bar{d}$ cross section and the $d \bar{d}$ cross section normalized to the $\nu \bar{\nu}$ cross section. See text for more detailed comments on these plots. 

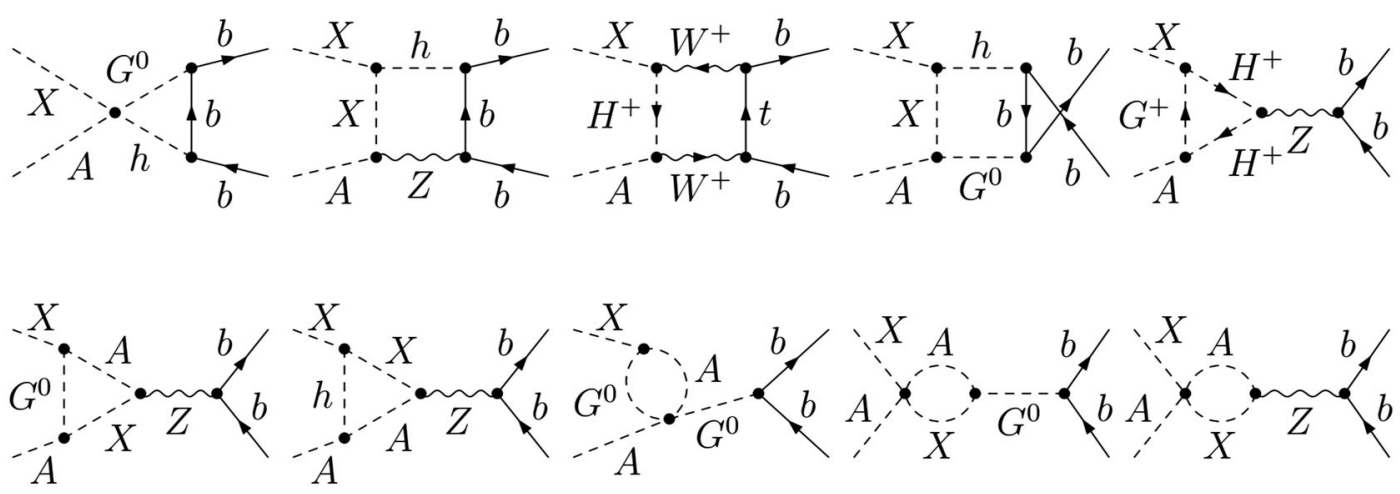

FIG. 3. Some one-loop diagrams (in the nonlinear Feynman gauge) for $A X \rightarrow b \bar{b}$. We only show a subset of the box corrections and the triangles. Note the last two diagrams involve the quartic coupling within the dark sector, $\lambda_{2}$. Unfortunately, the one mediated by the neutral Goldstone is proportional to the fermion mass and is therefore practically not sensitive to $\lambda_{2}$. The one mediated by the $Z$ will also give a contribution proportional to the fermion mass, since the one-loop integration will give, unlike the tree-level structure involving the difference between the two incoming momenta, a new Lorentz structure proportional to the $Z$ four momentum, which ends up giving a contribution proportional to the final fermion mass. Therefore, the $\lambda_{2}$ dependence only affects the $S$ wave.

effective cross sections defined solely in terms of physical observables,

$$
\begin{aligned}
\sigma_{v^{2}}^{f \bar{f}, \mathrm{eff}} \simeq \frac{12 \pi}{s} \frac{1-m_{-}^{2} m_{+}^{2} / s^{2}}{\left(1-M_{Z}^{2} / s\right)^{2}} \frac{\Gamma_{Z \rightarrow f f \bar{f}} \Gamma_{Z \rightarrow \nu \bar{\nu}}}{M_{Z}^{2}} \\
\sigma_{0}^{f \bar{f}, \mathrm{eff}} \simeq \frac{144 \pi}{s} \frac{m_{-}^{2} m_{+}^{2}}{s^{2}-m_{-}^{2} m_{+}^{2}} \frac{1}{\left(1-M_{Z}^{2} / s\right)^{2}} \frac{\Gamma_{Z \rightarrow \nu \bar{\nu}}^{2}}{M_{Z}^{2}} .
\end{aligned}
$$

First, $g^{2} / c_{W}^{2}$ in $(2.3)$ is traded for $\Gamma(Z \rightarrow \nu \bar{\nu})$. Then all partial widths in Eq. (2.4) are one-loop corrected partial widths. The results of the almost velocity independent corrections are shown in Table II. The effective corrective factor misses only about $-0.5 \%$ for the benchmark point P58 (almost independent of the nature of the fermion) of the full one-loop corrections and about $-1 \%$ for P60. These small corrections are the effect of the box contributions. These effective approximations are therefore excellent.

TABLE II. Full results for all channels for coannihilations into fermions given as percentage corrections. The approximate effective corrections, which corresponds to the effective cross sections in Eq. (2.4), are also given. The corrections are (practically) $v$ independent. While a close inspection of the $b \bar{b}$ channel reveals a very small presence of the $S$-wave contribution at $v \sim 0$, the $\lambda_{2}$ (and consequently) the $v$ dependence are too tiny. The $\lambda_{2} / v$ dependence is magnified in Fig. 4.

\begin{tabular}{lccc}
\hline \hline $\begin{array}{l}\text { Final } \\
\text { state }\end{array}$ & $\begin{array}{c}\text { P58: Full } \\
\text { correction }\end{array}$ & $\begin{array}{c}\text { P60: Full } \\
\text { correction }\end{array}$ & $\begin{array}{c}\text { Effective } \\
\text { approximation }\end{array}$ \\
\hline$l^{+} l^{-}$ & 5.90 & 5.21 & 6.49 \\
$\nu_{l} \bar{\nu}_{l}$ & 6.92 & 6.27 & 7.50 \\
$u \bar{u}$ & 4.95 & 4.24 & 5.54 \\
$d \bar{d}$ & 5.60 & 4.91 & 6.18 \\
$b \bar{b}$ & 4.05 & 3.01 & 4.54 \\
\hline \hline
\end{tabular}

The very tiny contribution of the $S$ wave at very small velocity (where the $P$ wave vanishes) is theoretically interesting even if it is without any phenomenological impact. It could have been larger if the fermion masses were larger. Theoretically, the $S$ wave is sensitive to $\lambda_{2}$ as explained in the caption of Fig. 3. We verified this property directly in the coannihilation into neutrinos, where no $\lambda_{2}$ dependence was detected numerically, and we confirmed that the largest $\lambda_{2}$ dependence occurs with the $b$ final state. Unfortunately, the maximum correction from the dark sector is at the level of 2 per-mille for $v=0$, where the cross section is smallest. As soon as the $P$-wave contribution kicks in, the tiny $\lambda_{2}$ dependence in the $S$-wave contribution is totally swamped, such that the one-loop

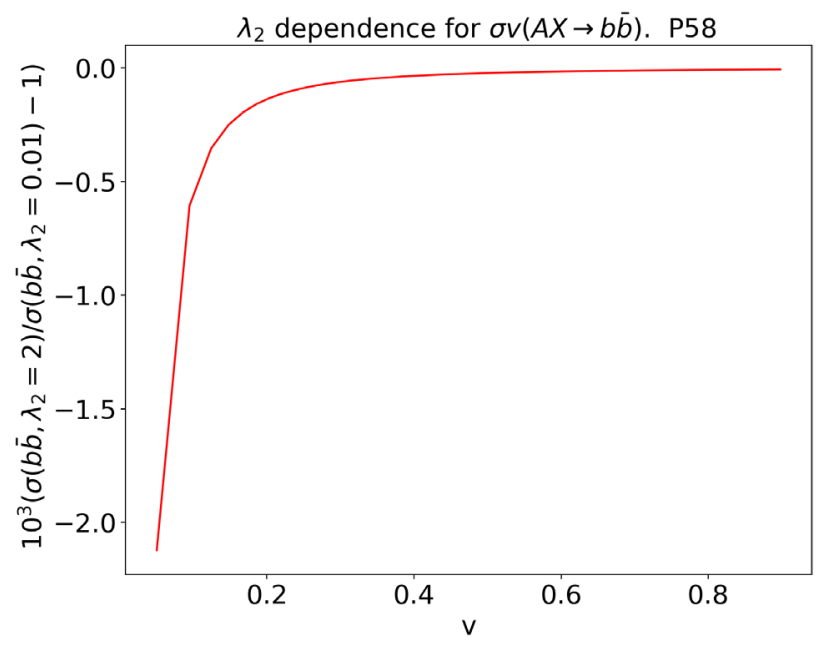

FIG. 4. The tiny $\lambda_{2}$ dependence in the $b \bar{b}$ coannihilation channel. The $\lambda_{2}$ dependence is measured relative to the $d \bar{d}$ cross section, where the $\lambda_{2}$ dependence is vanishing. Observe that the units in the graph indicate a variation which, at its highest, does not cross a couple of per-mille. 


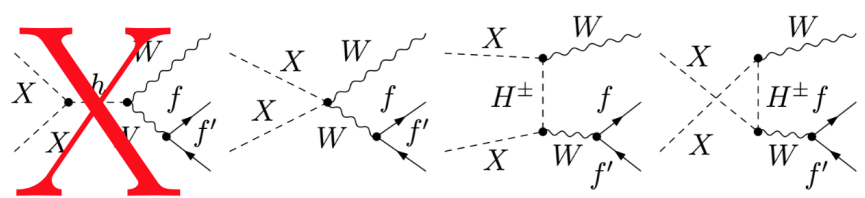

FIG. 5. A selection of diagrams for the tree-level cross section $X X \rightarrow W f \bar{f}^{\prime}$. Since $\lambda_{L}=0$ for the benchmark point P60, Higgs exchange does not take place for P60.

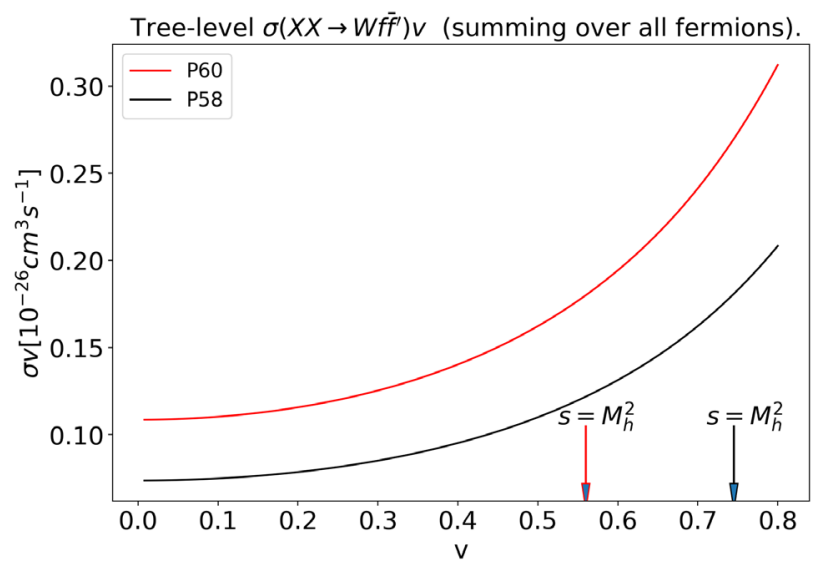

FIG. 6. The tree-level cross section $X X \rightarrow W f \bar{f}^{\prime}$ for the benchmark points $\mathrm{P} 58$ and $\mathrm{P} 60$. The arrow at $v \sim$ $0.56(0.745)$ indicates the position corresponding to the Higgs resonance (if crossed) for $M_{X}=60(58) \mathrm{GeV}$.

corrected cross section is, for all purposes, insensitive to $\lambda_{2}$. This very tiny $\lambda_{2}$ dependence is shown in Fig. 4 where we track the $\lambda_{2}$ dependence by considering the ratio in the $b \bar{b}$ cross section between $\lambda_{2}=0.01$ and $\lambda_{2}=2$.

\section{THE $X X \rightarrow W W^{\star}$ IN THE COANNIHILATION REGION}

\section{A. Tree-level considerations}

For P60, $X X$ annihilation to $W W$ below threshold with one $W$ decaying into a fermion pair, accounts, at tree level, for $10 \%$ of the relic density. This cross section does not suffer from the Boltzmann suppression. A nonzero value of $\lambda_{L}$ would have added a Higgs exchange contribution as we show in Fig. 5 in the $W W$ channel and in the $b \bar{b}$ channel $(X X \stackrel{h}{\rightarrow} b \bar{b})$.

As $v$ increases, the phase space for $W W^{\star}$ increases, and therefore, the cross section increases as Fig. 6 shows. Note that this cross section when compared to $A X \rightarrow f \bar{f}$ is far smaller (the latter will be reduced considerably by the Boltzmann factor). This increase with velocity is smooth across the Higgs resonance, $v=0.56$, a threshold that, as expected, is not felt since $\lambda_{L}=0$.

\section{B. One-loop results}

While $\lambda_{L}=0$ at tree level for $X X \rightarrow W f f^{\prime}$ with no SM Higgs exchange contributing, a renormalization of $\lambda_{L}$ is still called for in this process. This is because in general the $X X \rightarrow W W$ amplitude does depend parametrically on $\lambda_{L}$. As Fig. 7 clearly shows, an induced one-loop $h X X$ is generated, which entails a one-loop Higgs exchange contribution. The critical question is whether this will lead to an instability when the pole at $s=M_{h}^{2}$ is reached. Do we then have to, and how to, include a width? Casting doubt on the organization of the perturbation series? This same crucial point is dealt with in detail when we study cases where the resonance is present already at "tree level." In the

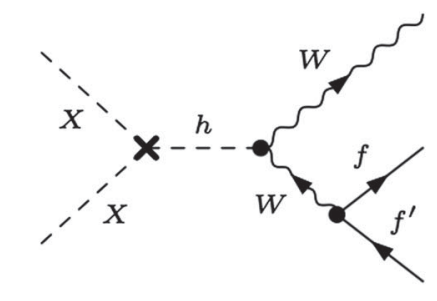

Counterterm Higgs vertex, $\lambda_{2}$

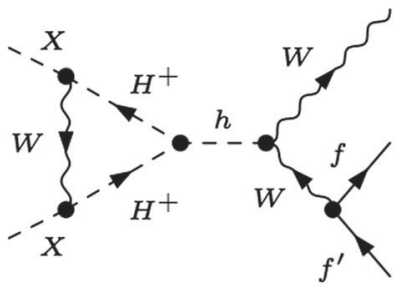

Higgs vertex

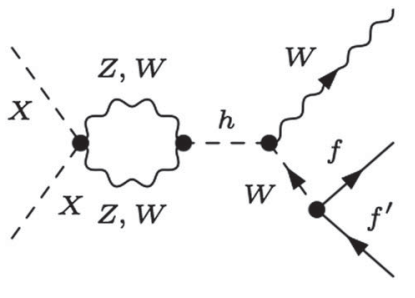

Higgs vertex

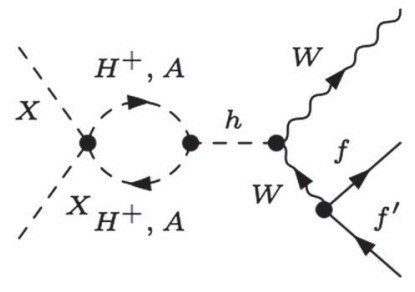

Higgs vertex, $\lambda_{2}$

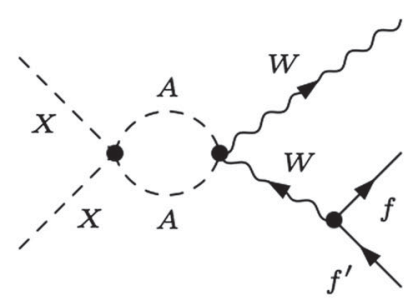

Box, $\lambda_{2}$

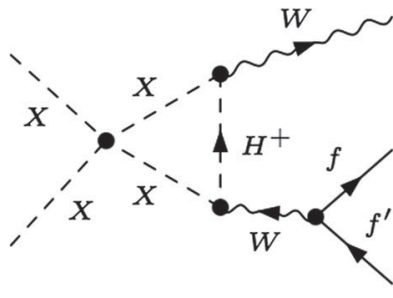

Box, $\lambda_{2}$

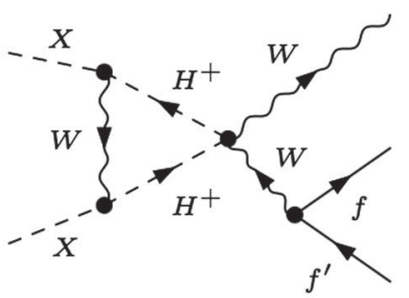

Box, rescattering

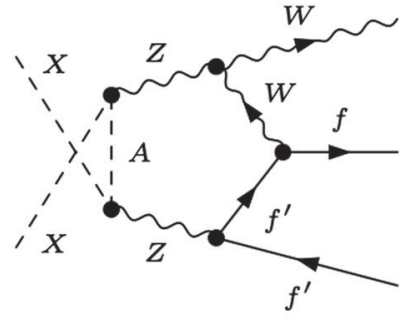

Pentagon

FIG. 7. A very small selection of the diagrams that enter the one-loop calculations of $X X \rightarrow W W^{\star}$. We highlight in particular Higgs exchange through the induced $h X X$ vertex at one-loop. We have also picked configurations where a $\lambda_{2}$ contribution shows up. These are part of what constitutes rescattering in the dark sector before annihilation into SM particles. An example of a pentagon diagram, which can not be considered as a factorized, $X X \rightarrow W W^{\star} \rightarrow W f f^{\prime}$ is also singled out (five-point function). 


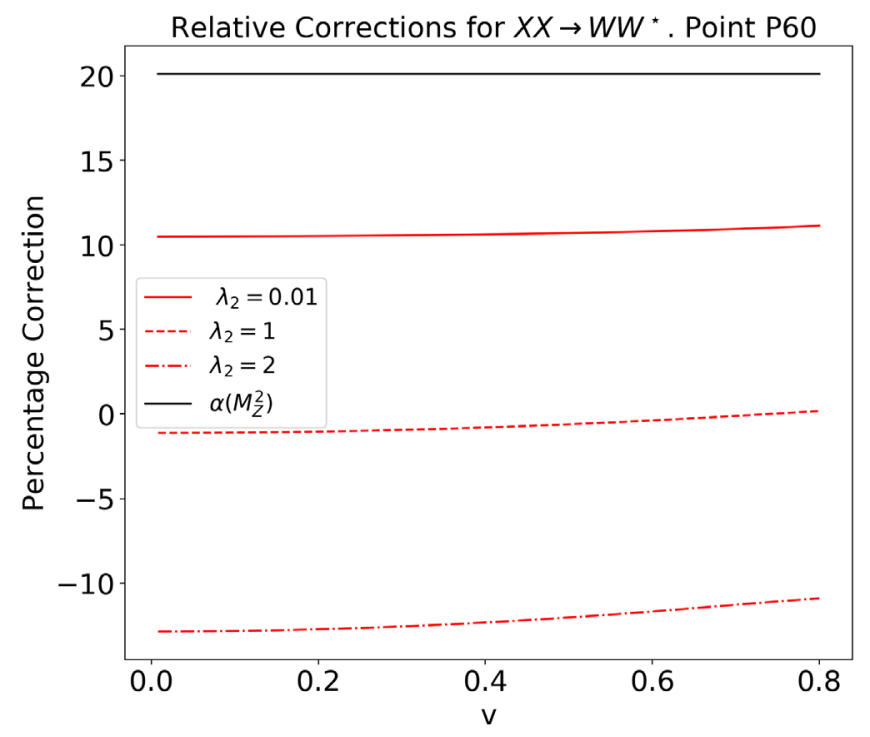

FIG. 8. The relative one-loop contribution to $X X \rightarrow W f f^{\prime}$ as a function of the relative velocity $v$ for $\lambda_{2}=0.01,1,2$. These corrections are compared to the effect of using an effective treelevel correction with $\alpha\left(M_{Z}^{2}\right)$.

present case, $\lambda_{L}=0$, there is no need to include a width. Since $h \rightarrow X X$ is open, the OS scheme is most appropriate for a definition and renormalization of $\lambda_{L}$. The OS scheme means that we trade $\lambda_{L}$ with $\Gamma_{h \rightarrow X X}$. In this particular case, the input translates into $\Gamma_{h \rightarrow X X}=0$. But $\Gamma_{h \rightarrow X X}=0$ is maintained at all orders entails, in fact, that the renormalized amplitude for $\mathcal{M}_{h \rightarrow X X}(s)=0$ at the renormalization point $s=M_{h}^{2}, s$ is the invariant mass of the $X X$ system. For a selection of one-loop contributions that make the $h \rightarrow X X$ vertex amplitude, see Fig. 7.

Consequently, $\left.\mathcal{M}_{h \rightarrow X X}(s) \frac{1}{s-M_{h}^{2}}\right|_{s=M_{h}^{2}}$ is continuous and the Laurent's series is well defined without a regulator, the width. There is then no pole structure as such and away from $s=M_{h}^{2}$ other (nonresonant) structures in the amplitude contribute as importantly. What is very interesting is that the one-loop amplitude is now $\lambda_{2}$ dependent; see Fig. 7. Note that $X X \rightarrow W W^{\star}$ is an abuse of language since such an off shell amplitude is not an element of the $S$ matrix. At one loop, this statement is supported by the appearance of essential pentagon contributions, where a split into a $W W^{\star}$ part is not possible. Nonetheless, the fact that the final state fermions are massless, we find no flavor dependence in the normalized (with respect to the tree-level) loop corrections when the full one-loop contribution is taken into account. In fine, this is reminiscent and strongly related to the fact that (normalized) electroweak corrections to $W$ decay into fermion pairs are flavor independent [10].

Our results for the electroweak corrections in this channel are displayed in Fig. 8. The behavior with respect to the velocity is very smooth, for all values of $\lambda_{2}$, confirming that the location of the Higgs boson is crossed continuously.

The $\lambda_{2}$ dependence at one loop is important. The full one-loop correction is about $10 \%$ for the smallest of $\lambda_{2}$, $\lambda_{2}=0.01$, it gives an almost vanishing total correction for $\lambda_{2}=1$ and then decreases to about $-10 \%$ for $\lambda_{2}=2$. For this value, $\lambda_{2}=2$, there is a $30 \%$ difference with the use of an effective $\alpha\left(M_{Z}^{2}\right)$. Therefore, $\alpha\left(M_{Z}^{2}\right)$ does not perform well as an approximation. The velocity dependence of the $\lambda_{2}$ correction is very weak. To summarize, at the level of the cross sections, the $\lambda_{2}$ contribution is important and discriminating. Will this conclusion still hold when we call these cross sections to compute the relic density considering that the weight of this channel is only $10 \%$ ?

\section{EFFECT ON THE RELIC DENSITY}

In order to translate the cross sections into a prediction on the relic density, we interface our calculations of the

TABLE III. The relic density for P58 and P60 with cross sections computed at tree level and after including the full electroweak corrections, as well as implementing the effective cross sections for $A X \rightarrow f \bar{f}$ and the cross sections calculated with $\alpha\left(M_{Z}^{2}\right)$. The percentage correction to the relic density is given in parenthesis. The percentage weight of each channel is computed for each implementation of the corrected cross section.

\begin{tabular}{|c|c|c|c|c|}
\hline & & $\Omega h^{2}$ & $A X \rightarrow f \bar{f}$ & $W W^{*}$ \\
\hline \multirow[t]{4}{*}{ P58 } & tree & 0.113 & $95 \%$ & $5 \%$ \\
\hline & One loop $A X \rightarrow f \bar{f}$ and tree $X X \rightarrow W W^{\star}$ & $0.108(-4.05 \%)$ & $95 \%$ & $5 \%$ \\
\hline & Effective $A X \rightarrow f \bar{f}$ and tree $X X \rightarrow W W^{\star}$ & $0.108(-4.45 \%)$ & $95 \%$ & $5 \%$ \\
\hline & $\alpha\left(M_{Z}^{2}\right)$ & $0.101(-10.62 \%)$ & $95 \%$ & $5 \%$ \\
\hline \multirow[t]{9}{*}{$P 60$} & tree & 0.116 & $91 \%$ & $9 \%$ \\
\hline & Full one loop, $\lambda_{2}=0.01$ & $0.111(-4.04 \%)$ & $91 \%$ & $9 \%$ \\
\hline & Full one loop, $\lambda_{2}=1$ & $0.112(-3.43 \%)$ & $91 \%$ & $9 \%$ \\
\hline & Full one loop, $\lambda_{2}=2$ & $0.113(-2.81 \%)$ & $91 \%$ & $9 \%$ \\
\hline & Effective $A X \rightarrow f \bar{f}$ and tree $X X \rightarrow W W^{\star}$ & $0.111(-4.36 \%)$ & $91 \%$ & $8 \%$ \\
\hline & Effective $A X \rightarrow f \bar{f}$ and loop $X X \rightarrow W W^{\star}, \lambda_{2}=0.01$ & $0.110(-4.92 \%)$ & $91 \%$ & $9 \%$ \\
\hline & Effective $A X \rightarrow f \bar{f}$ and loop $X X \rightarrow W W^{\star}, \lambda_{2}=1$ & $0.111(-4.32 \%)$ & $91 \%$ & $9 \%$ \\
\hline & Effective $A X \rightarrow f \bar{f}$ and loop $X X \rightarrow W W^{\star}, \lambda_{2}=2$ & $0.112(-3.71 \%)$ & $91 \%$ & $9 \%$ \\
\hline & $\alpha\left(M_{Z}^{2}\right)$ & $0.103(-11.21 \%)$ & $91 \%$ & $9 \%$ \\
\hline
\end{tabular}


one-loop corrected cross section with micrOMEGAs [11] as explained in [1]. Table III shows that the correction to the relic density for these low mass coannihilation configurations are small with a correction in line with what we found for the dominant cross sections $A X \rightarrow f \bar{f}$ (remember that $\Omega \propto 1 / \sigma$ ), which are practically $v$ independent. As expected, the corrections are much smaller than those found with the approximation of using a running $\alpha$. Since in P58 the contribution of the $W W^{\star}$ channel is less than about 5\%, we do not include the effect of the electroweak corrections in this channel. However, we include these corrections for P60 since this channel accounts for almost $10 \%$ of the total relic density (at tree level). While any small $\lambda_{2}$ dependence (which occurs at the very small $v$ in $A X \rightarrow f \bar{f}$ ) is washed out when the corrections are translated into the relic density calculation, the $\lambda_{2}$ dependence observed in the cross sections $X X \rightarrow W f \bar{f}^{\prime}$, make their way into the relic density even if the $W W^{\star}$ channel account for only $\sim 10 \%$. For $\lambda_{2}=2$, the correction in the $W W^{\star}$ channel counterbalances part of the correction from the contributions $A X \rightarrow f \bar{f}$ leading to an overall correction on the relic density less than $3 \%$. As a summary, in the coannihilation region, if one is satisfied with a calculation of the relic density with an accuracy no better than 5\%, one should content oneself with a tree-level calculation. An "improved" $\left[\alpha\left(M_{Z}^{2}\right)\right]$ gives a correction of about $10 \%$. The effective cross sections that are based on reexpressing the $A X \rightarrow f \bar{f}^{\prime}$ in terms of the one-loop partial decay widths of the $Z \rightarrow f \bar{f}$ reproduce the full one-loop results within $1 \%$.

\section{CONCLUSIONS}

We studied two IDM low mass DM benchmark points where coannihilation is the dominant contribution to the relic density. The coannihilation into fermions is driven essentially by the SM gauge coupling. The relative corrections are small. We show that they can be parametrized by simple effective cross sections involving $Z$ observables. The fermion flavor dependence is small. The small contribution from $W W^{\star}$ to the relic density is interesting since at one loop it reveals an important $\lambda_{2}$ dependence that accesses the purely dark sector. However, this $\lambda_{2}$ dependence gets diluted because of the small weight of $W W^{\star}$ to the relic density. Nonetheless, our calculations show that performing a full one-loop calculation gives smaller corrections than using an effective $\alpha\left(M_{Z}^{2}\right)$ into tree-level cross sections.

\section{ACKNOWLEDGMENTS}

We thank Alexander Pukhov for helpful discussions. H. S. is supported by the National Natural Science Foundation of China (Grants No. 12075043 and No. 11675033). He warmly thanks the CPTGA and LAPTh for support during his visit to France when this work was initiated. S. B. is grateful for the support received from IPPP, Durham, UK, where most of this work was performed and is also grateful to the support received from LAPTh where this work was initiated. N. C. is financially supported by IISc (Indian Institute of Science, Bangalore, India) through the C. V. Raman postdoctoral fellowship. He also acknowledges the support received from DST, India, under grant number IFA19-PH237 (INSPIRE Faculty Award).
[1] S. Banerjee, F. Boudjema, N. Chakrabarty, and H. Sun, preceding paper, Relic density of dark matter in the inert doublet model beyond leading order for the low mass region: 1. Renormalisation and constraints, Phys. Rev. D 104, 075002 (2021).

[2] N. G. Deshpande and E. Ma, Pattern of symmetry breaking with two Higgs doublets, Phys. Rev. D 18, 2574 (1978).

[3] R. Barbieri, L. J. Hall, and V.S. Rychkov, Improved naturalness with a heavy Higgs: An alternative road to LHC physics, Phys. Rev. D 74, 015007 (2006).

[4] A. Datta, N. Ganguly, N. Khan, and S. Rakshit, Exploring collider signatures of the inert Higgs doublet model, Phys. Rev. D 95, 015017 (2017),

[5] A. Belyaev, G. Cacciapaglia, I. P. Ivanov, F. Rojas-Abatte, and M. Thomas, Anatomy of the inert two Higgs doublet model in the light of the LHC and non-LHC dark matter searches, Phys. Rev. D 97, 035011 (2018).

[6] S. Banerjee, F. Boudjema, N. Chakrabarty, and H. Sun, following paper, Relic density of dark matter in the inert doublet model beyond leading order for the low mass region: 3. Annihilation in 3-body final state, Phys. Rev. D 104, 075004 (2021).

[7] S. Banerjee, F. Boudjema, N. Chakrabarty, and H. Sun, this issue, Relic density of dark matter in the inert doublet model beyond leading order for the low mass region: 4 . The Higgs resonance region, Phys. Rev. D 104, 075005 (2021).

[8] F. Boudjema and E. Chopin, Double Higgs production at the linear colliders and the probing of the Higgs selfcoupling, Z. Phys. C 73, 85 (1996).

[9] N. Baro, F. Boudjema, and A. Semenov, Full one-loop corrections to the relic density in the MSSM: A few examples, Phys. Lett. B 660, 550 (2008).

[10] D. Bardin, S. Riemann, and T. Riemann, Electroweak one loop corrections to the decay of the charged vector boson, Z. Phys. C 32, 121 (1986).

[11] G. Belanger, F. Boudjema, A. Goudelis, A. Pukhov, and B. Zaldivar, micromegas5.0: Freeze-in, Comput. Phys. Commun. 231, 173 (2018). 